\title{
Erratum to: Local Hölder continuity of weak solutions for an anisotropic elliptic equation
}

\author{
Agnese Di Castro
}

\section{Erratum to: Nonlinear Differ. Equ. Appl. DOI 10.1007/s00030-012-0160-7}

We found a gap in the proof of Lemma 4.2 (precisely in the contradiction argument to handle the level set estimates) that we are not able to overcome at present. For this, the main result presented in the manuscript cannot be obtained.

We believe that Hölder continuity holds true for bounded weak solutions but it could require a different strategy to be proved in such anisotropic framework.

Agnese Di Castro

Dipartimento di Matematica

Università degli Studi di Parma

Parco Area delle Scienze, 53/A

43124 Parma

Italy

e-mail: agnese.dicastro@unipr.it;

agnese.dicastro@gmail.com

Agnese Di Castro

CMUC

Department of Mathematics

University of Coimbra

Apartado 3008

3001-454 Coimbra

Portugal

The online version of the original article can be found under doi:10.1007/s00030-012-0160-7. 